\title{
El modelo conceptual en los sistemas de procesamiento de la información
}

\author{
Miguel-Ángel López Alonso \\ Departamento de Biblioteconomía y Documentación \\ Universidad Carlos III de Madrid
}

\subsection{Resumen}

Se analiza la sustitución progresiva de los modelos convencionales del proceso de recuperación de la información por modelos cognitivos que aplican el modelo humano de memoria y aprendizaje a los agentes inteligentes de recuperación de la información. Se profundiza en la aplicación de la teoría cognitiva al análisis contextual de manera que las respuestas se valoren con un nuevo concepto de relevancia global centrado en los usuarios. A continuación se proponen ontologías para la estructuración de las bases de conocimientos, como potentes útiles conceptuales para la extracción, compilación y recuperación del conocimiento. Se concluye con propuestas de cómo esta evolución hacia el paradigma cognitivo se plasmará en las bibliotecas digitales del futuro. (Autor)

Palabras clave: Modelo cognitivo. Usuario. Relevancia. Ontología. .

\subsection{Abstract}

The substitution of the classical physical model of information retrieval by cognitive models is studied, considering carefully its implications for the design of intelligent information retrieval agents. The application of cognitive theories to contextual analysis is considered, and it is proposed that retrieval results should be evaluated from a wider and user-centred concept of relevance. Therefore, some ontologies for setting up knowledge bases are introduced as powerful conceptual tools for the extraction, compilation and retrieval of knowledge. Finally, some proposals for assisting the evolution toward a cognitive paradigm in the future Digital Libraries are given. (Author amended).

Keywords: Cognitive model. User. Relevance. Ontology. 


\section{Introducción}

Estableceremos como hipótesis que la Ciencia de la Documentación viene adoptando desde comienzos de los ochenta una tendencia creciente al abandono de la visión de la información como objeto físico o soporte y a su sustitución por un enfoque centrado en el individuo como usuario final.

Tomando la Teoría Cognitiva como área interdisciplinar del entorno de la Sociedad de la Información, su modelo sustenta un enfoque subjetivo y postula que la información es siempre "información para alguien", y que el usuario de los sistemas de información está obligado a jugar su papel. De acuerdo con sus postulados, las cosas son únicamente informativas para un usuario, si son nuevas, comprensibles y relevantes, es decir, si cambian la estructura previa de su conocimiento respecto de ellas (Ingwersen, 1992).

\section{Premisas}

a) Según su metodología, el objetivo fundamental de los Sistemas Conceptuales es "optimizar el acceso intelectual del usuario a la información". Cuando ocurre como en la actualidad que, debido a los avances en las tecnologías de la información, los problemas de acceso físico dejan de ser apremiantes, les sustituyen los derivados del acceso intelectual que se convierten ahora en los determinantes de dichos sistemas. Los usuarios dejan de ser contemplados como meros utilizadores de información, y los sistemas electrónicos tienen que resolver sus problemas de recuperación del conocimiento subyacente en la información, referentes a:

- La sobresaturación de materiales informativos.

- La coordinación de sus sistemas personales con los sistemas externos.

- La identificación de la información más relevante para sus necesidades, etc.

b) Tradicionalmente los objetos informativos se han analizado con referencia a unas teorías y unos principios del sistema de información considerados como inmutables. Pero, como el uso de la información depende de una situación previa que determina tanto las necesidades de información como el carácter y la organización de sus fuentes más relevantes, la doctrina se está decantando hacia el procesamiento situacional, pragmático y específico de la información, con respecto al área del conocimiento abarcada y al nivel de conocimientos del usuario (Hjorland ; Albrechtsen, 1995). 


\section{Tesis}

Para aplicar esta modelo cognitivo dentro del contexto de la persona, la sociedad y la cultura:

1) Se precisará que las respuestas a cada problema personal se orienten a través del análisis estadístico, los agentes inteligentes o el razonamiento automático, y que sus resultados se valoren con un nuevo concepto de relevancia global que nos lleve a un nuevo modelo integrado de la información.

2) Se necesitarán útiles más sofisticados para la extracción, compilación y recuperación del conocimiento de entre las ingentes cantidades de datos disponibles.

3) Se requerirá la creación de sistemas altamente integrados que procesen la información tanto en el puesto de trabajo individual como dentro de las instituciones.

\subsection{EI análisis contextual y la relevancia global desde el punto de vista del usuario}

Partiendo de que dicho modelo cognitivo considera el análisis documental como una situación de rutina cognitiva, se precisa la normalización de la metodología de éste último para obtener:

- Un análisis contextual profundo de los documentos que forman el conocimiento colectivo de las organizaciones.

- Que resuelva el problema de la gestión de los documentos electrónicos como una decisión estratégica, pensando en su posterior integración dentro de la WWW.

Esta normalización debe permitirnos:

a) Plantear las ecuaciones de búsqueda en forma de necesidad de información para obtener documentos "relevantes".

b) Ampliar la "cultura informativa" de los usuarios sobre las fuentes de información de su interés y la forma de utilizarlas.

Para ello, a pesar de los avances logrados en la mejora de los ratios de efectividad en la recuperación de la información desde la interacción entre el usuario y el sistema, algunos autores como Bates (1986) y Beghtol (1995) insisten en profundizar en el estudio de la estructura y la organización de la información para una valoración adecuada de los resultados de las recuperaciones, dados los problemas de indeterminación derivados de la flexibilidad y creatividad del lenguaje natural. De la misma opinión se muestran Molto (1993) y Moreiro (1993) al 
propugnar el perfeccionamiento en la selección de los conceptos de cada documento mediante el análisis semántico y discursivo del contenido textual.

Estamos plenamente de acuerdo con ellos en que, para evitar la indeterminación intrínseca a dicho lenguaje, debe ahondarse en el análisis del contenido, tanto desde un nivel microestructural o inductivo (de abajo-arriba) como desde un nivel global o deductivo (de arriba-abajo), que abarque la macroestructura o tema general sobre el que trata cada texto. $\mathrm{Al}$ analizar las macroestructuras parciales de cada texto, se avanza en la comprensión profunda del texto, como conocimiento específico que debe confirmar la macroestructura global. Según Beghtol (1995), este proceso tendrá lugar durante la lectura profunda del texto y será como de "controlado olvido", gobernado por macroreglas que "dejen en suspenso" momentáneamente la información menos importante, con objeto de que aparezca claramente identificado el tema principal del texto.

El estudio de la relevancia, a pesar de ser un tema dominante desde los años setenta, se ha enfocado desde dos orientaciones bien diferentes: la centrada en el sistema o la centrada en el usuario, sin que todavía en la actualidad se haya llegado a un consenso definitivo.

Con la localización de los recursos documentales mediante la navegación hipertextual en redes, han surgido serias dudas sobre el mantenimiento de las medidas cuantitativas tradicionales de la relevancia. Parece que los problemas que afectan a la valoración de la relevancia hipertextual tienen poco que ver con el modelo experimental de Cranfield, pensado inicialmente para medir la relevancia de elementos aislados en una reducida base documental. La necesidad de desarrollo de "modelos diferentes" para la evaluación y diseño de los nuevos instrumentos de recuperación de la información resulta innegable.

Los modelos que representan la opinión de los usuarios propugnan la naturaleza subjetiva de la relevancia y son más complejos que los modelos centrados en el sistema, ya que deben asimilar la información de las distintas fuentes para realizar su elección a través de la correspondencia de sus tres campos de actividad:

- El físico, que trata de la acción realizada.

- El afectivo, que trata de las opiniones expresadas, y ...

- El cognitivo, que trata de los conceptos del contenido y del proceso (Kuhlthu,1991).

El complejo fenómeno de la relevancia abarca relaciones a muy distintos niveles:

1) Jerárquicas, estructurales o de equivalencia, típicas del desarrollo de los lenguajes documentales. 
2) Entre el lenguaje del texto y la capacidad lingüística del usuario, o entre el contenido sustancial del texto y la amplitud del conocimiento básico del usuario.

3) Entre el tema del pasaje del texto y el tema demandado por el usuario ("topic matching relations", más complejas que las habituales relaciones de equivalencia temática desde el punto de vista del sistema (Paice, 1991).

Mientras que los aspectos relacionados con el campo físico se investigan dentro del campo más amplio de las relaciones sistema usuario, que tratan los interfases interactivos. Los otros dos campos: el afectivo y el cognitivo, deben concretarse dentro de las investigaciones sobre la relevancia psicológica que desarrollan los diferentes sistemas de gestión de la información (Harter, 1992).

Unas medidas de relevancia ampliamente aceptadas deben considerar a los documentos y a las ecuaciones de búsqueda como "unidades interrelacionadas", con el objetivo final puesto en que las recuperaciones sean útiles al usuario (Dominich, 1994). Por ello, demanda el desarrollo de un nuevo Modelo Conceptual Integrado de la Información que, tome como punto de partida la "interacción total" y considere los documentos y las preguntas como entidades del mismo tipo, pero, que no considere a la relevancia como una entidad subjetiva, sino dependiente de ambas entidades.

Se están abriendo camino los sistemas de procesamiento de la información en línea que adoptan técnicas cualitativas de medición de la eficiencia de las recuperaciones muy próximas a las hipótesis sobre este "nuevo tipo de relevancia" (propuesto por Dominich), de tomar en consideración las opiniones del usuario en cuanto a los documentos recuperados (Swanson, 1986).

Estos sistemas centran el estudio de la relevancia de las recuperaciones en:

a) La evaluación de la utilidad de la información obtenida por el usuario para la resolución de su problema.

b) La integración de los resultados, en las sucesivas y más depuradas ecuaciones de búsqueda, con vistas a obtener la mayor sinergia sistema usuario.

Estas técnicas miden la eficiencia del sistema con dos medidas cualitativas: la exactitud (que recuerda a la precisión de los sistemas convencionales) y la integridad (que evoca a la exhaustividad de los sistemas convencionales).

Dado que la medición de la relevancia es un fenómeno imprescindible para la completa comprensión del proceso cognitivo del comportamiento humano en la Organización de la Información, la profundización deberá partir de la conside- 
ración de su naturaleza como un concepto multidimensional, dinámico y complejo, pero, medible (Schamber, 1994).

A partir de este nuevo concepto de relevancia, para el que la recuperación necesita entender tanto la naturaleza de los sistemas de información como las necesidades y los requerimientos de relevancia del usuario, se proponen métodos para la evaluación de dichos sistemas integrados que tomen en consideración la naturaleza dinámica de las necesidades de información, variable en el tiempo para un mismo usuario (construcciones mentales dinámicas) (Borlund e Ingwersen, 1985) y relacionada estrechamente con la visión de la teoría cognitiva sobre una recuperación de la información situacional, pragmática y específica.

\section{Ontologías para la estructuración de las bases de conocimientos y de sus tesauros autogenerados internos.}

El usuario necesita sistemas de recuperación de la información que utilicen potentes estructuras del conocimiento, capaces de analizar su pregunta, compararla con los documentos de la base de datos, e identificar los fragmentos de éstos que resultan relevantes para su búsqueda. Parte de la información documental disponible en un espacio conceptual, no se recupera si no existe un vocabulario controlado, basado en alguna clasificación conceptual (tesauros, encabezamientos de materias o sistemas de clasificaciones), que induzca relaciones asociativas con diversos apartados de los textos y extraiga las respuestas específicas.

Una ontología que pueda ser utilizada con una base de conocimientos debe:

1) Proporcionar una metavisión de la estructura y de la terminología del dominio que facilite las recuperaciones automáticas (Schamber, 1990).

2) Mantener su relación con el contexto en que fue creada (a partir del análisis contextual de los documentos de una determinada base de datos) que le permita diferenciar entre la base de conocimientos y las reglas de decisión para la resolución de problemas de un agente experto.

3) Incluir las normas para redefinir o cambiar los conceptos, de manera que resuelvan los problemas del contexto en uso (tareas y no requerimientos funcionales) (Vanwelkenhuysen y Mizoguchi, 995), evolucionando de manera permanente.

En lo que se ha dado en llamar "el esfuerzo para compartir" diferentes bases de conocimientos utilizando las tecnologías de la Inteligencia Artificial, se estudia fundir las categorías de varios sistemas ontológicos de manera que se integren y se comuniquen entre sí. Para la representación de su conocimiento se perfila un consenso a favor de un modelo trilateral de tres esquemas enlazados:

1) El esquema ontológico o estructura semántica central. 
2) El esquema de representación, que abarca las interacciones externas con los demás elementos del sistema, sus representaciones gráficas o simbólicas y los "puntos de vista" del usuario.

3) El esquema de aplicación, que trata de la manera en que el sistema se integra en un ordenador o red determinada (las llamadas reglas del núcleo del sistema o agente experto).

Otra de las herramientas propuestas para evitar la falta de precisión del lenguaje natural, trata de la incorporación en un Tesauro Conceptual (autogenerado internamente en la base de conocimientos) de los conceptos obtenidos del Análisis de Contenido de los documentos que facilite las recuperaciones desde el interfase sistema usuario. De esta forma se incrementa la variedad de las ecuaciones de búsqueda del usuario, o al menos le muestra la variedad de las existentes; lo que, de acuerdo con el Principio de Variedad de Requisitos de Ashby, incrementa simultáneamente la cantidad de respuestas del sistema consultado.

Los Tesauros Conceptuales, diseñados para ayudar en la enunciación de las preguntas en la Recuperación de la Información en grandes bases de datos, propuestos por autores como Bates (1990), Lancaster (1986) o Schmitz-Esser (1991), palian la indeterminación de las búsquedas en lenguaje natural, especialmente en aquellas bases de datos cuyos documentos con texto completo no han sido previamente indizados con ningún otro tesauro.

Diversos experimentos en los que se apoya a los usuarios en la enunciación de sus ecuaciones de búsqueda con términos adicionales extraídos de un Tesauro Conceptual (Croft y Das, 1990; Kristensen, 1993), han llegado a doblar la precisión en el número de documentos recuperados si el usuario selecciona y usa los términos sugeridos como adicionales a sus propios términos (Ekmekcioglu, Robertson y Willet, 1992). Según otros que tratan de medir los efectos de la ampliación de las búsquedas mediante este tipo de tesauros, los mejores resultados se obtienen cuando los usuarios:

a) Pueden elegir entre una gran cantidad de términos del campo específico tratado, sean suyos, de la Base de Datos o incluso de indizaciones previas.

b) Controlan interactivamente el proceso de navegación por el tesauro, en vez de utilizar procedimientos automáticos (Jones, 1995).

\section{El espacio conceptual y los sistemas hipertextuales de redes neuronales para el desarrollo de sistemas altamente integrados}

Dado que las bases documentales no se diseñan para la toma de decisiones directamente, la organización de sus conocimientos requiere del análisis contex- 
tual de cada uno de sus documentos y del resumen de sus notas más destacadas mediante dos fases consecutivas:

$\left.1^{a}\right)$ La extracción previa de los datos menos relevantes o metaclasificación de los documentos.

$\left.2^{a}\right)$ El posterior examen especializado, para estructurar el conocimiento referido a los temas específicos del área de conocimientos abarcada en una determinada ontología.

Algunas investigaciones han propuesto la separación de los resultados de las dos fases anteriores en una estructura para la organización del conocimiento o espacio de indización (red semántica), y en otra para la organización del contenido informacional o espacio de contenidos (red de documentos). Esta distinción facilitaría la recuperación de la información e induciría enlaces dinámicos para un mantenimiento independiente de ambas redes.

Las redes semánticas son redes asociativas en las que las unidades conceptuales representadas por nodos son meras entidades semánticas y las relaciones representadas por enlaces son asociaciones de entidades. Dewèze formaliza la representación de las relaciones semánticas con la adopción de una teoría semántica extralexical que sitúa a un nivel superior al de los lenguajes naturales, en la perspectiva de construir tesauros multilingües. En esta teoría, un significado es definido como "un conjunto de semas a los que se pueden atribuir relaciones lexicales en varios idiomas posteriormente" (Dewèze, 1981, p. 68).

La Red Semántica Conceptual facilita:

- El encaminamiento de las hojas hacia una raíz y de una raíz hacia las hojas, gracias a la función de orientación presentada por las clases de discriminantes o facetas.

- La transición de un árbol hacia otro a través de los nudos polijerárquicos o relaciones asociativas.

- La extensión a un árbol completo o la restricción a un subárbol o incluso la restricción a árboles parciales.

- La exploración transversal de la red al buscar configuraciones correspondientes a una o varias facetas en una reunión o en una intersección de árboles.

Sus propiedades generales son:

a) La independencia respecto de las relaciones léxicas.

b) La facilidad de extensión o de encaminamiento hacia otros conceptos, a partir de cualquier nodo de la red. 
c) La posibilidad del multilingüismo, al entrar en la red semántica por el léxico de una lengua cualquiera, mediante la fijación de relaciones léxicas en varios idiomas, y ...

d) El tiempo de acceso reducido, sea cual sea el punto de entrada en la red, debido a la facilidad de seguimiento de las referencias cruzadas.

Si partimos de que los sistemas hipertextuales son todavía la mejor herramienta para mantener relaciones no lineales entre documentos y para relacionar los descriptores de los tesauros y los códigos clasificatorios, mientras que la organización de la información contenida en los trabajos impresos está dispuesta secuencialmente (en capítulos y párrafos con una variedad de índices), la información contenida en las bases de datos a texto completo precisa de cierta estructuración que permita una recuperación no tan rígida (mediante referencias cruzadas entre sus documentos asociados).

Los sistemas que aplican el modelo cognitivo a la manera en que las personas piensan los problemas más complejos, se organizan esencialmente como una red semántica de conceptos unidos mediante relaciones no lineales. Su organización hipertextual permite moverse a través de una red no lineal de macroestructuras de conceptos, definidos a partir de las asociaciones de ideas del usuario, derivadas de su nivel de conocimientos de cada disciplina y de su filosofía personal. Esta aproximación cognitiva permite que cualquier persona utilice el modelo mental aplicando metáforas (nuevas estructuras cognitivas) autogeneradas dentro del contexto de cada aplicación, mientras que la implementación misma puede basarse en el modelo semántico general. Tal aproximación facilita la navegación y el análisis de la base de datos en estudio, independientemente de la aplicación específica y de los modelos mentales diferentes de cada usuario (Carlson y Gonzales, 1993).

3.1. Limitaciones para el desarrollo de los sistemas hipertextuales.

a) La desorientación de los usuarios para encontrar documentos relevantes durante su navegación entre los textos de las bases documentales muy extensas. La unión de la información de todas las fuentes disponibles en un número pequeño de nodos, conduce a una sobresaturación de enlaces en dichos nodos. La introducción de muchos subenlaces dificulta la navegación e incrementa las posibilidades de perderse en el espacio hipertextual, además, reduce el deseo de los usuarios de explorar la información, como consecuencia de una "situación de fracaso" cognitivo que condiciona su reacción personal ante ella (Winograd y Flores, 1986).

A medida que se vayan perfeccionando los Agentes Expertos del campo de la Inteligencia Artificial, se irán perfeccionando las Bases de

Scire. 4 : 1 (en.-jun. 1998) 29-43. 
Conocimientos de los Sistemas Integrados de Procesamiento de la Información, facilitando su viabilidad como herramientas globales para la organización y recuperación del conocimiento en la actividad diaria profesional, apoyados en dicha tecnología hipertextual integrada en las redes neuronales artificiales (Doszkocs, 1990).

Estas últimas proporcionan un mapa de las variables introducidas en la búsqueda y de las obtenidas en la recuperación, y son capaces de almacenar información de forma distribuida, aprendiendo de los problemas reales tras un período de validación que actualice las respuestas (Berger y Van Bommel, 1997). Dado que algunas de las características de los sistemas de procesamiento de la información requieren capacidades avanzadas de predicción, las redes neuronales pueden demostrar su potencialidad en el aprendizaje de los comportamientos de los usuarios a lo largo de un determinado período para predecir sus futuras necesidades, tratadas como un problema de series no lineales en el tiempo.

b) La necesidad de controlar manualmente los enlaces hipertextuales creados durante el trabajo automático, dado que éstos no contienen información conceptual directa sobre el contenido de los documentos relacionados. Dichos sistemas no pueden procesar dinámicamente la información existente en sus documentos, recuperando solamente aquellos que tienen relaciones predefinidas con un nodo principal o mantienen relaciones dinámicas con otros documentos.

Para su correcto aprovechamiento, los sistemas de gestión de la información documental deberán contar con interfases hombre-máquina, capaces de especificar las relaciones entre los conceptos a partir de las ontologías preestablecidas. Aquí las redes neuronales pueden actuar como filtro que minimice la entrada de datos, colaborando con las herramientas de extracción en el interfase de usuario (ontología hipertextual, tesauro conceptual, etc.). El usuario deberá tener acceso a ellos a través de una representación gráfica (tabla de contenidos gráficos, con sus conexiones visibles mediante enlaces hipertextuales), por la que pueda rastrear la red semántica de conceptos del área en estudio y la red de documentos de la base de datos, siguiendo su línea de interés.

\section{Conclusiones e influencia en las bibliotecas digitales del futuro.}

Un modelo conceptual de gestión de la información que parta de un enfoque centrado en el trabajo habitual del usuario final, debe incluir potentes estructuras de representación del conocimiento, capaces de recoger todo tipo de relaciones, 
sus participantes y sus interacciones, e interactuar con el usuario a través de "interfases inteligentes" durante el planteamiento de las ecuaciones de búsqueda.

Dado que esta relación entre los ordenadores y los usuarios es el principal problema para la integración de los atributos de la inteligencia humana y la tecnología, se estima que la recuperación de la información necesita de una estrecha colaboración entre el usuario final y los programas informáticos de los agentes inteligentes de la IA, utilizados en los Sistemas Integrados de Recuperación de la Información. Esto deberá lograrse mediante el desarrollo de potentes interfases, capaces de compartir la experiencia y la alta fidelidad de los canales de comunicación del usuario con el poder de procesamiento y la alta velocidad de los canales de comunicación de la teleinformática (Marchionini, 1992).

1) El procesamiento de la información se desplaza desde el contenido de la información hacia el estudio de los procedimientos utilizados para su tratamiento, de manera que la oferta sea plural, el funcionamiento se someta a reglas éticas y se evalúe plenamente la calidad.

Para la obtención de un diseño centrado en la filosofía del usuario, se deberán analizar y describir las necesidades concretas de éste que permitan especificar a continuación las tareas que realizará el sistema:

a) La interpretación del Lenguaje Natural de las ecuaciones de búsqueda del usuario, que puede contener términos lingüísticos poco precisos o ambiguos, difíciles de traducir a su lenguaje documental,

b) la localización de los documentos en la Base de Datos, que puede seguir la llamada "manipulación directa" con diversos modos de interacción manual, y la llamada "inferencia plausible" con reglas declarativas y la especificación del modo de visualización, de manipulación y de aplicación de los datos en el motor de inferencia de los agentes expertos,

c) la presentación de los resultados al usuario en la forma que un consultor humano actúa en la resolución de problemas, a partir del procesamiento del Lenguaje Natural a distintos niveles y del reconocimiento de las Estructuras de Representación del Discurso, como apoyatura teórica de sus funciones "inteligentes" en los diferentes tipos de menús.

2) La evolución del papel de las bibliotecas como consecuencia de la manipulación masiva de información digitalizada multimedia (imágenes, vídeo y sonido), y del cambio de su papel de archivo de información por el de directorio de distribución de fuentes, ha motivado que la naturaleza del acceso a la información se haya redefinido como "un acceso intelec- 
tual o cognitivo" (Griffin, 1998), según lo que el usuario demanda en cada momento y no conforme a lo poseído por cada biblioteca.

Este acceso intelectual deberá ser investigado desde tres grandes áreas:

$1^{a}$ ) La representación de las actividades humanas.

Para entender a los usuarios y sus conocimientos, necesitamos representar éstos últimos y las necesidades de información de los primeros de acuerdo con determinados principios de estructuración del conocimiento, estrategias para la solución de problemas, organización de la información y estrategias para la búsqueda.

$2^{\mathrm{a}}$ ) La representación de los contenidos y de las colecciones.

Para conocer que es importante en la representación del conocimiento, necesitamos conocer previamente los problemas del usuario y sus esquemas mentales. Ambos componentes, las necesidades de información de los usuarios y las estructuras para la representación del conocimiento, deben estar centrados en el primero e integrarse y depender uno de otro, según dicho nuevo modelo de comunicación tomado del paradigma conceptual.

$3^{\text {a) }}$ La representación de los componentes de los sistemas.

Este acceso intelectual necesita de sistemas de información capaces de manipular fragmentos de los documentos para extender la síntesis del conocimiento y, a la vez, procesar y mostrar los resultados, creando nuevo conocimiento (Chaumier, 1986).

La investigación futura deberá encaminarse a la búsqueda de interfases "inteligentes", con los usuarios como destinatarios principales, dada la necesidad de desarrollos avanzados para interactuar con la información. Para evitar que se pierda la oportunidad de diseñar sistemas en perfecta armonía con las preferencias de los usuarios, sus interfases deberán tener las siguientes características mínimas:

a) Ser "verdaderamente distribuidos", de modo que, independientemente de su localización, sean capaces de modificar (en tiempo real) los diferentes caminos de acceso a los datos suministrados, por cualquier usuario, en cualquier lugar y en cualquier tipo de formato (Belkin, 1987, p. 407).

b) Aceptar la "manipulación directa" durante el rastreo sofisticado de los documentos, mediante la realización de las siguientes tareas "inteligentes": 
- Una continua representación de los objetos de interés.

- La facilitación de las acciones físicas por medio del uso de distintas herramientas: teclado, ratón, joystick, pantalla sensible, etc.

- Operaciones rápidas, incrementales y reversibles cuyo impacto en el objeto de interés sea inmediatamente visible, y

- Un aprendizaje progresivo, por etapas, que pueda ser utilizado con conocimientos mínimos previos (Schneiderman, 1986, p. 57).

c) Proporcionar "ayuda continuada", a través de un diálogo interactivo con estrategias alternativas de búsqueda, mediante las siguientes operaciones concatenadas:

- El análisis de las preguntas del usuario,

- la identificación de sus necesidades de información y su fácil conversión en preguntas,

- la entrega al usuario de los resultados de forma clara y concisa, y

- la sugerencia de alternativas para búsquedas más ajustadas (Turtle, 1992).

d) Integrar diferentes mecanismos de precisión: bases de conocimientos, tesauros, etc., aplicaciones y medios de difusión, con la finalidad de que los usuarios se comuniquen entre sí o accedan a diferentes Bases de Datos. Y utilizar diferentes servicios globales en línea, mediante nuevas formas más naturales, intuitivas y flexibles, en el marco general de un Modelo Conceptual Integrado de la Información (Ingwersen, 1996, p. 34).

e) Describir en sus interfases las estructuras estratégicas de control del grado de implicación del usuario en las búsquedas, y el tipo de capacidades exigibles al sistema (Bates, 1990, p. 577).

3) Las bibliotecas digitales pueden provocar la redefinición de la biblioteca como institución, y aportar su apoyo a un nuevo modelo de comunicación interactivo compartido con los usuarios.

4) Los nuevos profesionales de la información deberán ser poseedores de conocimientos interdisciplinares psicosociológicos, capaces de abarcar la interdisciplinariedad de la Ciencia de la Información demandada por el modelo cognitivo, en el entorno altamente cambiante de la Sociedad de la Información. 


\section{Referencias}

Bates, M. J. (1986). Subject Access in Online Catalogs: A Design Model. // Journal of ASIS. 37 : 6 (1986) 357-376.

Bates, M. J. (1990). Where should the person stop and the information search interface start?. // Information Processing Management. 26 : 5 (1990) 577.

Beghtol, C. (1995). Facets as interdisciplinary undiscovered public knowledge. // Journal of Documentation, 51 : 3 (1995) 194-224.

Belkin, N. J. et al. (1987). Distributed Expert-Based Information Systems: An Interdisciplinary Approach. // Information Processing Management. 23 : 5 (1987) 407.

Berger, F. C . ; Van Bommel, P. (1997). Augmenting a characterization network with semantic information. // Information Processing and Management. 33 : 4 (1997) 453479.

Borlund, P. ; Ingwersen, P. (1997). The development of a method for the evaluation of interactive information retrieval systems. // Journal of Documentation, $53: 3$ (1997) 225-250.

Carlson, P. ; Gonzales, G. (1993). The knowledge board: Using hypertext as an intelligent workspace for writing issues-based prose. // Journal of educational multimedia and hypermedia. 2 : 14 (1993) 417-431.

Croft, W. B. ; Das, R. (1990). Experiments with query acquisition and use in document retrieval systems. // Proceeedings of the 13th Conference on Research and Development in Information Retrieval. Brussels, Belgium, 9/1990,

Chaumier, J. (1986). Análisis y Lenguajes Documentales. El tratamiento lingüístico de la información documental. Barcelona : Mitre, 1986.

Dewèze, A. (1981). Réseaux sémantiques: essai de modélisation; aplication à l'indexation et à la recherche documentaire. Lyon : Universidad Claude Bernard, 1981. Tesis doctoral.

Dominich, S.(1994). Interaction Information Retrieval. // Journal of Documentation. 50 : 3 (1994) 197-212.

Doszkocs, T. E. ; et al. (1990). Connectionist Models and Information Retrieval. // ARIST. (1990) 209-260.

Ekmekcioglu, F. C. ; Robertson, A. M. ; Willet, P. (1992). Effectiveness of query expansion in ranked-output document retrieval systems. // Journal of Information Science. 18 (1992) 139-147.

Griffin, S.M. (1998). Taking the Initiative for Digital Libraries. // The Electronic Library. $16: 1$ (1998) 24-27.

Harter, S. P. (1992). Psychological Relevance and Information Science. // Journal of ASIS. $43: 9$ (1992) 602-615.

Hjorland, B. ; Albrechtsen, H. Toward a New Horizon in Information Science: Domain Analysis. // Journal of the ASIS. (May, 1995) 26.

Ingwersen, P. (1992). Information Retrieval Interaction. London : Taylor Graham, 1992. 
Ingwersen, P. (1996). Cognitive Perspectives of Information Retrieval Interactions: elements of a cognitive IR Theory. // Journal of Documentation. 52 : 1 (1996).

Jones, Susan ; et al. (1995). Interactive Thesaurus Navigation: Intelligence Rules Ok?. // Journal of the American Society for Information Science. 46 : 1 (1995) 58-59.

Kristensen, J. (1993). Expanded End-user's Query statements for free text searching with a search-aid thesaurus. // Information Processing \& Management. 29 : 6 (1993) 733744.

Kuhlthu, C. C. (1991). Inside the Search Process: Information Seeking from the User's Perspective. // Journal of the ASIS. 42 : 5 (1991) 362.

Lancaster, F. W. (1986). Vocabulary control for information retrieval. Information Resource Press, 1986. 270 p.

Marchionini, G. (1992). Interfaces for End-User Information Seeking. // Journal of the ASIS. $43: 2$ (1992) 156.

Molto, M. (1993). Improving full text search performance through textual analysis. // Information Processing Management. (1993) 615-632.

Moreiro, J. A. (1993). Aplicación de las Ciencias del Texto al Resumen Documental. Madrid: U. Carlos III-BOE, 1993.

Paice, Chris D. (1991). A Thesaural Model of Information Retrieval. // Information Processing Management. 27 : 5 (1991) 435.

Schamber, L. ; et al. (1990). A re-examination of relevance: toward a dynamic, situational definition. // Information Processing Management. 26 (1990) 755-775.

Schamber, L. (1994). Relevance and Information Behavior. // ARIST. 29 (1994) 3-48.

Schmitz-Esser, W. (1991). New Approaches in Thesaurus Application. // International Classification, $18: 3$ (1991) 143-147.

Shneiderman, B. (1986). Designing menu selection systems. // Journal of the ASIS. $37: 2$ (1986) 57.

Swanson, D. R. (1986). Subjetive versus objective relevance in bibliographic retrieval systems. // Library Quarterly. 56 (1986) 389-398.

Turtle, H. R. ; Croft, W. B. (1992). A comparison of text retrieval models. // The Computer Journal. 35 : 3 (1992) 279-290.

Vanwelkenhuysen, J.; Mizoguchi, R. (1995). Ontologies and Guidelines for Modeling Digital Systems. // Proceedings of 9th Knowledge Acquisition for KBS Workshop. Banff: Canada, marzo 1995.

Winograd, T.; Flores, C.F. (1986). Understanding computers and cognition. Norwood, NJ : Addison-Wesley, 1986. 